\title{
DIRECT ELECTRICAL STIMULATION PROMOTES GROWTH AND ENHANCES SURVIVAL OF ANEUROGENIC MUSCLES OF THE CHICK EMBRYO ${ }^{1}$
}

\author{
JOSEPH W. BLOOM, JANE BUTLER, JOYCE BRIERLEY, AND ETHEL COSMOS ${ }^{2}$ \\ Department of Neurosciences, McMaster University Medical Centre, Hamilton, Ontario, Canada L8N 3Z5
}

Received April 26, 1984; Revised July 2, 1984; Accepted August 1, 1984

\begin{abstract}
Analyses of embryonic aneurogenic muscles indicate that several processes associated with early myogenesis in vivo proceed in the absence of peripheral nerves. However, aneurogenic muscles demonstrate impaired growth and limited survival. To investigate whether neurally mediated activity is responsible for these phenomena, aneurogenic muscles of the chick embryo were directly stimulated in vivo via implanted electrodes. Volumetric analyses of stimulated aneurogenic brachial (latissimus dorsi) muscles from Stage (St) 33 (7.5 to 8 days) through St 37 (11 days) demonstrated that growth was enhanced significantly beyond the level characteristic of unstimulated aneurogenic muscles. Moreover, for the majority of embryos, the stimulation regimen actually rescued the posterior latissimus dorsi muscle which characteristically does not survive beyond St 32 (7.5 days) in the aneurogenic state. Thus, our results implicate activity per se as an important factor necessary for the proper growth and survival of brachial muscles during early embryogenesis. The stimulation regimen, however, did not alter myosin ATPase profiles.
\end{abstract}

The earliest period for detection of myosin ATPase activity in the brachial musculature of chick embryos is during the fourth day in ovo; by the end of the first week of embryogenesis, differentiation of distinct muscle fiber types has occurred (Butler and Cosmos, 1981a, b). Experiments designed to determine the role of neurally mediated influences on this event demonstrate that fiber type differentiation proceeds on schedule in aneurogenic brachial muscles, i.e., muscles which never experienced direct contact with peripheral nerves (Butler et al., 1980 , 1982a). Using a different aneurogenic model, the superior oblique muscle of the duck embryo, Sohal and colleagues have shown that several other important events of early myogenesis also occur independent of nerves, namely, the differentiation of acetylcholine receptors (Creazzo and Sohal, 1983) and high molecular weight acetylcholinesterase, as well as the formation of postsynaptic folds and basal lamina (Sohal and Wrenn, 1984). Even though, in vivo, many events associated with the incipient differentiation of embryonic muscles proceed in the absence of neuronal influences, embryonic aneurogenic muscles do exhibit impaired growth rates and, ultimately, limited survival in the chick (Butler et al., 1982a), salamander (Tweedle et al., 1974; Popiela, 1976, 1977), and duck (Sohal and Holt, 1980; Creazzo and Sohal, 1983).

Postembryonic studies concerned with the role of neurons

\footnotetext{
${ }^{1}$ We express our gratitude to E. Paul Allard for his technical assistance and for the final preparation of the photographic figures. This research was supported by grants awarded to E. C. by the Muscular Dystrophy Association, the Muscular Dystrophy Association of Canada, and the John A. Bauer Fund. J. W. B. was the recipient of a Muscular Dystrophy Association of Canada postdoctoral fellowship.

${ }^{2}$ To whom reprint requests should be addressed.
}

on the growth and maintenance of muscles identify two major mechanisms for investigation, namely, neurally evoked activity of the muscle and neurotrophic influences (for reviews, see Close, 1972; Jolesz and Sreter, 1981; McArdle, 1983). Experiments designed to study these factors in postembryonic animals have been concerned primarily with an alteration of pre-established phenotypes of muscles which have already experienced functional contacts with their own appropriate nerves. In contrast, the aneurogenic embryonic model, first introduced in 1927 by Hamburger, affords the unique opportunity to assess the effect of one specific nerve-associated function on a muscle which has never experienced contact with peripheral motor nerves. Using this model, we chose to investigate the role of activity on the growth and survival of embryonic muscles.

To investigate the effect of experimentally applied electrical stimulation on the growth and survival of aneurogenic brachial muscles, the appropriate segment of the neural tube was deleted at day 2 in ovo, prior to the outgrowth of nerves, and the muscles were then chronically stimulated via surgically implanted electrodes. Our results indicate that, in the absence of peripheral nerve contacts, direct electrical stimulation alone served to enhance significantly the growth and survival of aneurogenic muscles during early stages of myogenesis. Furthermore, their myosin ATPase profiles remained unaltered. A preliminary report of these experiments has been presented (Bloom et al., 1983).

\section{Materials and Methods \\ Surgical procedures}

Neural tube removal. Fertile White Leghorn eggs, obtained from a local hatchery (Martindale Farms), were incubated at $37.4^{\circ} \mathrm{C}$ and at a humidity of $56 \%$. At approximately 2 days in ovo, the eggs were 
windowed and the embryos were staged according to the morphological criteria of Hamburger and Hamilton (1951). For the majority of embryos, the neural tube opposite somites 13 to 23 inclusive was removed at Stage (St) 13 (48 to $52 \mathrm{hr} ; 19$ pairs of formed somites), as previously described (Butler et al., 1982a); however, a few embryos were at St 12 ( 45 to $49 \mathrm{hr} ; 16$ pairs of formed somites) or St 14 (50 to $53 \mathrm{hr} ; 22$ pairs of formed somites) at the time of surgery. Since the entire brachial neural tube segment was deleted prior to both the outgrowth of ventral root fibers (Castro, 1963; Butler et al., 1982a) and the formation of dorsal root ganglia (Romanoff, 1960), myogenic cells which would eventually contribute to the formation of the brachial musculature were rendered aneurogenic; i.e., they never experienced innervation. Following surgery, the eggs were resealed with cellophane tape and returned to the incubator. In total, 238 neural tube removals were performed for the present study with a postoperative mortality rate of $46 \%$. For the present experiments the term "aneurogenic embryo" refers to embryos subjected to deletion of the brachial segment only, not the entire neural tube.

Electrode implantation. On day 6 of incubation, the shell windows were enlarged and the embryos were examined. Embryos were selected for electrode implantation only if they appeared morphologically normal except for the mid-dorsal depression in the brachial region which marked the site of neural tube deletion. Embryos were rejected if they exhibited either gross abnormalities, such as spinal bifidas and microphthalmia $(n=32)$, or movement of the wings $(n=25)$. The absence of wing motility was a preliminary indication that the brachial musculature was aneurogenic since it has been demonstrated that motility is neurogenic in origin (for review, see Oppenheim, 1982).

All surgical instruments and electrodes were sterilized with $70 \%$ ethanol prior to implantation of the electrodes. To achieve direct stimulation of aneurogenic muscles, electrodes were positioned in the brachial region as follows. First, the chorioallantoic membrane was freed at its edge and deflected without damage to its circulation. Then, the amnion was removed as completely as possible without damaging the abdominal wall of the embryo. Once the embryo was exposed, it was stabilized dorsally with a small polyethylene brace to facilitate impalement with two $75-\mu \mathrm{m}$ silver wire uninsulated electrodes whose tips had been previously sharpened. Silver wires were used because platinum, although electrochemically preferable, was too stiff and, thus, more difficult to manipulate safely in the delicate embryonic tissues. One electrode was positioned in the carpal region of the right forewing. To achieve placement of the second electrode, it was first inserted into the brachial region ventrally, posterior to the right axilla, and passed dorsally between the scapula and the rib cage to emerge finally at the dorsal midline just anterior to the wings (Fig. 1). After impalement, the electrode tips protruding beyond the embryo were bent to prevent displacement of the electrodes and their wires were fixed to the eggshell with dental wax. The shell window was then sealed with a glass coverslip to permit subsequent observation of the embryo. Finally, microconnectors were soldered to the electrode wires and the eggs were returned to the incubator before final connections were made to the stimulator. The entire procedure for the preparation and impalement of the embryos lasted approximately $15 \mathrm{~min}$. Even though care was taken to avoid damage to the embryo, especially its blood vessels, the post-impalement mortality rate was $65 \%$, i.e., 37 of the 57 embryos subjected to this procedure did not survive.

\section{Stimulation protocol}

Direct stimulation of aneurogenic brachial muscles was accomplished with a Digitimer pattern generator driving an isolated Digitimer stimulator. Capacity-coupled monopolar stimulus pulses of $2 \mathrm{msec}$ duration were delivered at 100 pulses/sec in 100 -msec bursts. The bursts were repeated every $6 \mathrm{sec}, 24 \mathrm{hr} /$ day after the initiation of stimulation. 'I'he stimulation regimen was based in part on the frequency of wing movements observed with unoperated embryos (Cauwenbergs et al., 1983) and partly on effective patterns that did not damage the embryos. For most embryos, stimulation was begun on day 6 in ovo, immediately following impalement, but for a few it was not begun until early in day 7. Effective electrical stimulation caused movements of the brachial musculature; each burst of stimuli resulted in a single contraction. Electrode polarization progressively reduced the efficiency of stimulation with time. Therefore, embryos were examined through the glass window several times a day during the stimulation period, and the stimulus voltage was adjusted as necessary to maintain stimulus effectiveness. Stimulation was maintained for 2 to 5 days after impalement.

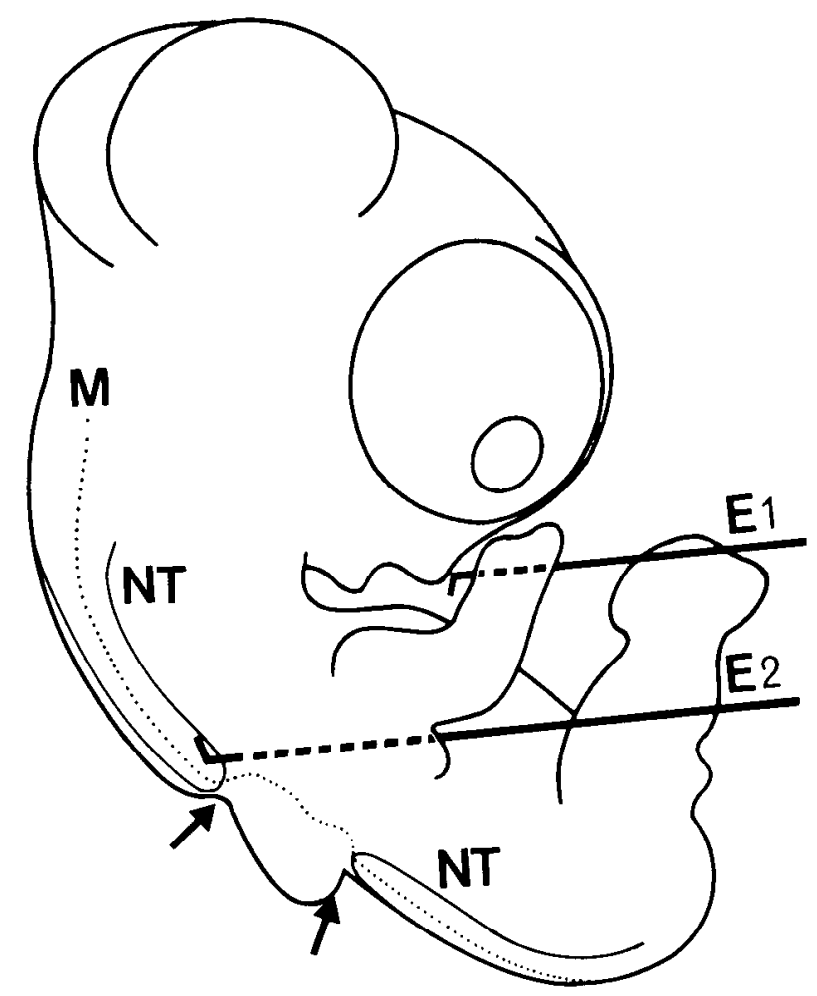

Figure 1. Diagram of a day 6 chick embryo to illustrate the position of the implanted electrode wires to achieve direct electrical stimulation of the right aneurogenic brachial area. The course of the electrodes through the embryo is indicated by the heavy dashed lines. The position and extent of the extirpated neural tube segment in the brachial region are also illustrated (arrows). $E 1$ and $E 2$, electrodes; $M$, dorsal midline; $N T$, neural tube.

\section{Control embryos}

Embryos subjected to deletion of the brachial neural tube but not impaled with electrodes served as primary controls for the stimulated aneurogenic embryos. These unstimulated aneurogenic embryos were drawn from two sources: those reported in an earlier study (Butler et al., 1982a) and those produced specifically for the present study. Since analyses of these two groups gave similar results, they were treated as a single pool of embryos and formed the base line for comparison of the effects of stimulation on aneurogenic muscles. Furthermore, data from both the stimulated $(n=17)$ and unstimulated $(n=18)$ aneurogenic preparations were compared to those of unoperated (innervated) $(n=20)$ embryos.

\section{Histochemical and volumetric analyses}

At the termination of the stimulation regimen, experimental embryos were removed from the egg and staged, and the electrode wires were carefully removed. The embryos were decapitated, eviscerated, and frozen for cryostat sectioning. Serial longitudinal sections of each embryo were analyzed by a silver-cholinesterase reaction to verify the aneurogenic status of the brachial muscles, i.e., the complete absence of nerves (Butler et al., 1982a), and by the myosin ATPase reaction, following alkali and acid preincubation, to determine muscle fiber types, as described previously (Butler and Cosmos, 1981a, b). Muscles deemed aneurogenic were subjected to volumetric analysis. Individual muscles were reconstructed with serial camera lucida drawings which were analyzed by a Zeiss Videoplan Image Analysis System. The anterior latissimus dorsi (ALD) and posterior latissimus dorsi (PLD) muscles were selected for quantitative analyses since these two distinctive muscle types had previously demonstrated differential responses to the aneurogenic condition (Butler et al., 1982a).

\section{Results}

\section{General observations}

Seventeen of the 57 embryos subjected to both deletion of the brachial neural tube at day 2 in ovo and electrical stimula- 
tion via implanted electrodes survived for analysis. These stimulated embryos, ranging in age from St 33 ( 7.5 to 8 days) through St 37 (11 days), were normal in size and general appearance except for the indentation in the spinal region where the brachial neural tube had been extirpated. Thus, neither the neural tube deletion nor the presence of electrode wires in the right shoulder and wing affected the general morphology of the forelimbs compared to that of unoperated embryos (Fig. 2).

To determine the effect of direct stimulation on the growth of aneurogenic muscles, the aneurogenic status of the brachial muscles had to be verified. Therefore, each of the 17 stimulated embryos were completely sectioned, and the presence or absence of nerves in the brachial musculature was assessed with the silver-cholinesterase histochemical reaction. Microscopic analyses determined that 14 of the 17 experimental embryos were indeed aneurogenic, and these were subjected to quantitative volumetric and muscle fiber type analysis. The silvercholinesterase reaction identified the presence of intramuscular axons in the three rejected embryos as well as the presence of fragments containing neuronal cell bodies within the area of neural tube deletion.

\section{Volumetric analyses}

Anterior latissimus dorsi muscle. To quantify the effect of chronic direct stimulation on the growth of aneurogenic brachial muscles, the total volumes $\left(\mu \mathrm{m}^{3} \times 10^{6}\right)$ of the ALD and PLD muscles were determined using a computerized Image Analysis System (Zeiss). These muscles were selected since our previous examination of aneurogenic embryos indicated that the latissimus dorsi muscles were especially sensitive to deprivation of motor innervation. Among the four muscles derived from the latissimus dorsi primordia, namely, the ALD, PLD, dorsocutaneous latissimus dorsi (DLD), and metapatagial latissimus dorsi (MLD), only the ALD muscle survived in the aneurogenic state beyond St 32 (7.5 days) (Butler et al., 1982a).

Figure 3 compares total muscle volumes of innervated ALD muscles to those of unstimulated aneurogenic and stimulated aneurogenic ALD muscles. Values for innervated (unoperated, $n=40)$ and unstimulated aneurogenic $(n=29)$ ALD muscles represent the mean value \pm SEM from embryos between St 30 (6.5 days) and St 37 (11 days), whereas the data for stimulated aneurogenic ALD muscles are plotted as individual values. As discussed below, the unstimulated aneurogenic values were obtained exclusively from embryos subjected only to the re- moval of the brachial neural tube. Eleven of the 14 stimulated aneurogenic ALD muscles analyzed demonstrated markedly enhanced growth compared to unstimulated aneurogenic ALD muscles. As indicated in Figure 3, externally applied electrical stimulation not only initially increased the limited, endogenous capacity for the growth characteristic of unstimulated aneurogenic ALD muscles, but it also promoted growth throughout the entire period analyzed. Furthermore, microscopic analysis demonstrated that the stimulated aneurogenic ALD muscles were well formed, compact muscles.

To explain the variability among the individual values for stimulated aneurogenic ALD muscles, especially the three values in Figure 3 which fall close to unstimulated aneural values, certain factors may be considered. First, although after implantation the electrode tips were bent and the electrode wires secured to the egg with dental wax, conceivably some displacement of the electrodes during the period of stimulation could occur as a result of vigorous total body movements characteristic of young embryos. In fact, the St 36 (10-day) value of 44 $\mu \mathrm{m}^{3} \times 10^{6}$ (Fig. 3 ) was obtained from an embryo whose electrodes were observed to be positioned superficially when the stimulation regimen was terminated. Second, the relationship of the electrodes to various muscle groups could change as the wings grow. Third, it was not technically feasible to detect whether specific individual muscles were being either uniformly or effectively activated in each individual preparation.

Despite the variable effect among individual embryos, the ability of direct electrical stimulation to promote growth of the aneurogenic ALD muscle is evident. Indeed, the positive response of aneurogenic ALD muscles to stimulation extended to the contralateral side in some experimental embryos. As indicated, values from the contralateral (left) side of the stimulated aneurogenic preparations were not included in Figure 3 since a comparison of these values with data derived from unstimulated aneurogenic embryos indicated that the field of electrical current had apparently spread to the left side in some preparations. At St 35 (8 to 9 days), the left aneurogenic ALD muscle of two of six stimulated embryos was affected; their total muscle volumes were 5 -fold greater than aneurogenic values. Likewise, at St 36 (10 days), growth of the left aneurogenic ALD muscle of two of four experimental embryos increased approximately 1- to 2 -fold.

Posterior latissimus dorsi muscle. The positive influence of electrical stimulation on the aneurogenic PLD muscle is especially striking. Characteristically, the PLD muscle of unstim-
Figure 2. Photograph of a St 36 (10-day) stimulated aneurogenic embryo. The implanted electrode wires protrude from the forewing $(E 1)$ and the ventral aspect of the shoulder region $(E 2)$. The gross morphology of the embryo, including the stimulated wing, is normal.

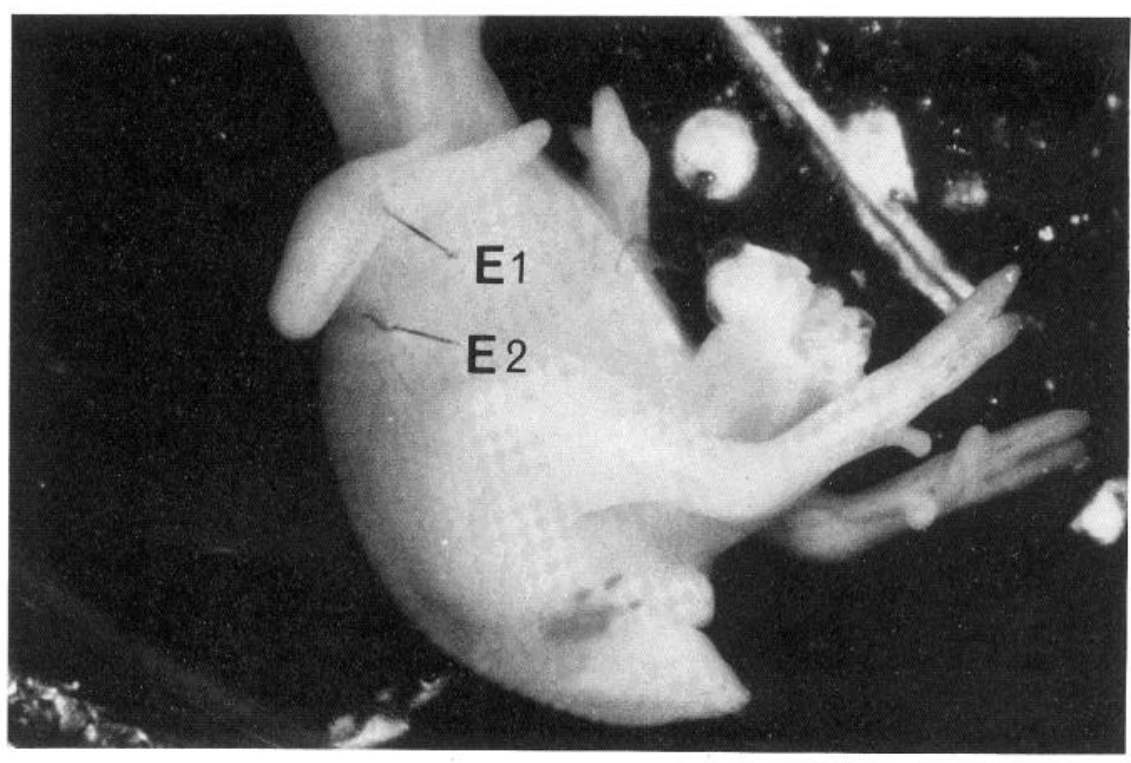




\section{ANTERIOR LATISSIMUS DORSI}

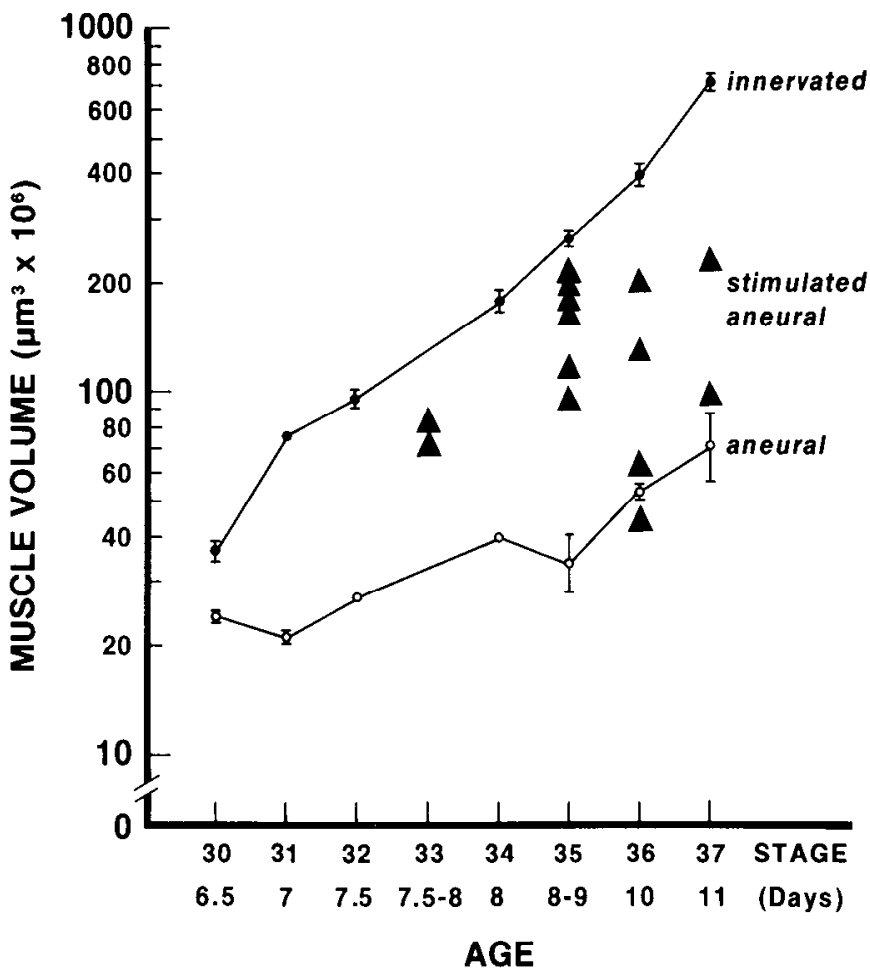

Figure 3. Comparative growth of aneural, stimulated aneural, and innervated (unoperated) ALD muscles from St 30 (6.5 days) through St 37 (11 days) in ovo. The total volume $\left(\mu \mathrm{m}^{3} \times 10^{6}\right)$ of each stimulated aneural ALD muscle is plotted separately, whereas the values for both unstimulated aneural and innervated muscles represent the mean vol ume \pm SEM.

ulated aneurogenic embryos fails to survive beyond St 32 (7.5 days) (Fig. 4). In contrast, direct chronic stimulation of the aneurogenic right wing from day 6 in ovo onward extended the survival of the aneurogenic PLD muscle. The right PLD muscle was rescued in 9 of the 14 stimulated aneurogenic embryos analyzed between St 33 ( 7.5 to 8 days) and St 37 (11 days). The contralateral aneurogenic PLD muscle was absent. Furthermore, significant growth of the rescued PLD muscle was observed in all preparations but one (Fig. 4). In addition to the PLD muscle, histochemical analyses indicated that the stimulation regimen also rescued the right DLD and MLD muscles. Similar to the PLD muscle, these two muscles also do not survive beyond St 32 ( 7.5 days) in the absence of peripheral motor nerves.

\section{Fiber type analyses}

Although previously we had demonstrated that differentiation of muscle fiber types, a process which normally begins during the first week of development in ovo (Butler and Cosmos, 1981a, b), is independent of neuronal influences, the possibility existed that experimentally applied electrical stimulation might alter this expression. The myosin ATPase profiles characteristic of aneurogenic and stimulated aneurogenic ALD and PLD muscles at St 35 ( 8 to 9 days) are presented in Figure 5, $a$ and $b$, and the stimulated aneurogenic muscles at St 37 (11 days) are represented in Figure 5c. All muscles demonstrate appropriate alkali-stable myosin ATPase reactions. Whereas the right PLD muscle is absent in the aneurogenic, unstimulated preparations (Fig. $5 a$ ), it is present and exhibits strong alkali stability in the aneurogenic but stimulated embryos (Fig. 5, b and $c$ ). Note also in Figure $5 c$ the presence of the right DLD muscle which, similar to the aneurogenic PLD muscle, was

\section{POSTERIOR LATISSIMUS DORSI}

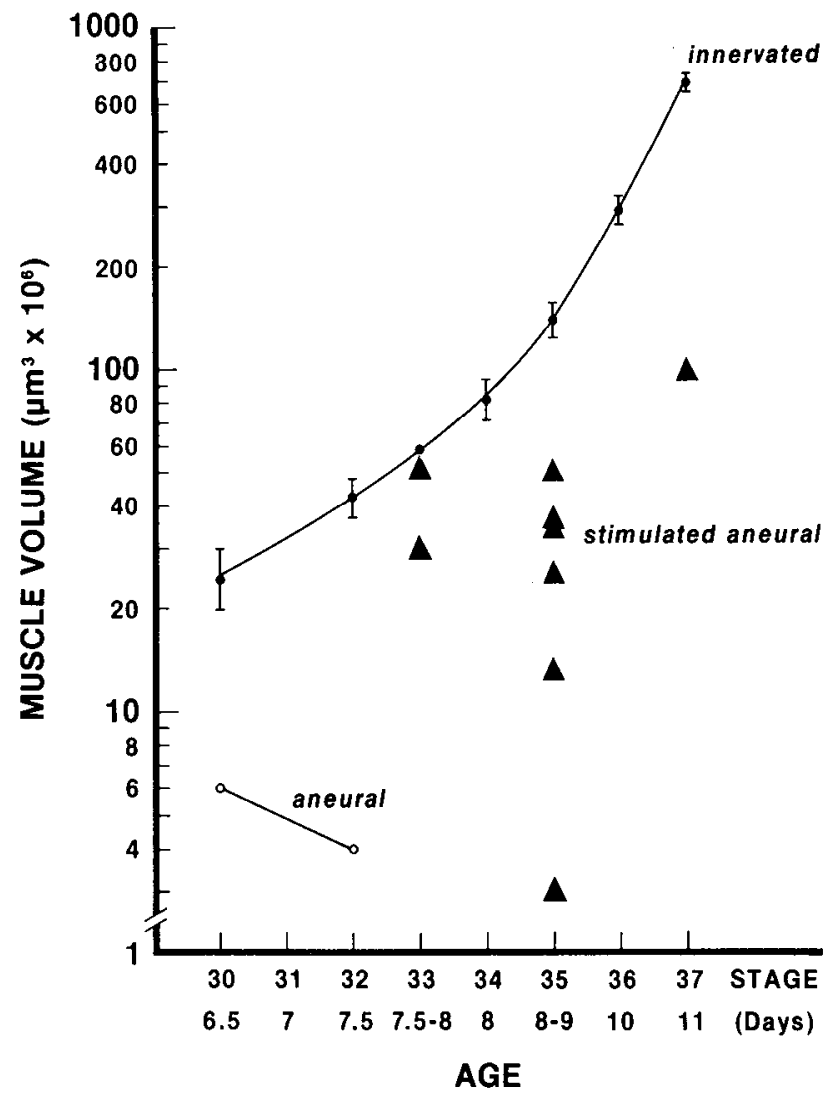

Figure 4. Comparative growth of aneural, stimulated aneural, and innervated (unoperated) PLD muscles from St 30 (6.5 days), through St 37 (11 days) in ovo. The total volume $\left(\mu \mathrm{m}^{3} \times 10^{6}\right)$ of each stimulated PLD muscle is plotted separately, whereas the values for innervated PLD musles represent the mean volume \pm SEM. Note that the aneural PLD muscle which is not stimulated fails to survive beyond St 32 (7.5 days).

rescued as a result of stimulation. The stimulated muscles also exhibit appropriate acid-stable myosin ATPase activity (Fig. 6). A comparison of Figure $6 a$ with its serial section in Figure $5 b$ indicates that the ALD muscles are both alkali and acid stable. Likewise, both the ALD and DLD muscles of the St 37 (11-day) preparation exhibit this dual stability.

As noted previously (Toutant et al., 1979; Butler and Cosmos, 1981b), within the unoperated PLD muscle from approximately St 37 (11 days) onwards, a distinct population of fibers appears that is both acid and alkali stable. The origin of these "atypical" PLD fibers, which initially increase numerically through St 41 (15 days) and then decrease, remains unknown. Although previously we were unable to determine whether the expression of this subpopulation of PLD fibers was dependent upon innervation since the aneurogenic PLD muscle did not survive beyond St 32 (7.5 days), we can now state that an intact nerve is not necessary for their appearance. At St 37 (11 days), the presence of these "atypical" PLD fibers is clearly evident within the stimulated aneurogenic PLD muscle (Fig. 6b); however, even at St 35 ( 8 to 9 days), a few fibers within the stimulated aneurogenic PLD muscle are beginning to demonstrate a faint acid-stable reaction (Fig. $6 a$ ).

Since innervated (unoperated) ALD, PLD, and DLD muscles at the same stages demonstrate similar profiles (Butler and Cosmos, 1981b; Butler et al., 1982a), the stimulation protocol which functioned to rescue the aneurogenic PLD muscle and to enhance growth of the aneurogenic ALD muscle did not alter myosin ATPase profiles. The presence of the scapula in all 

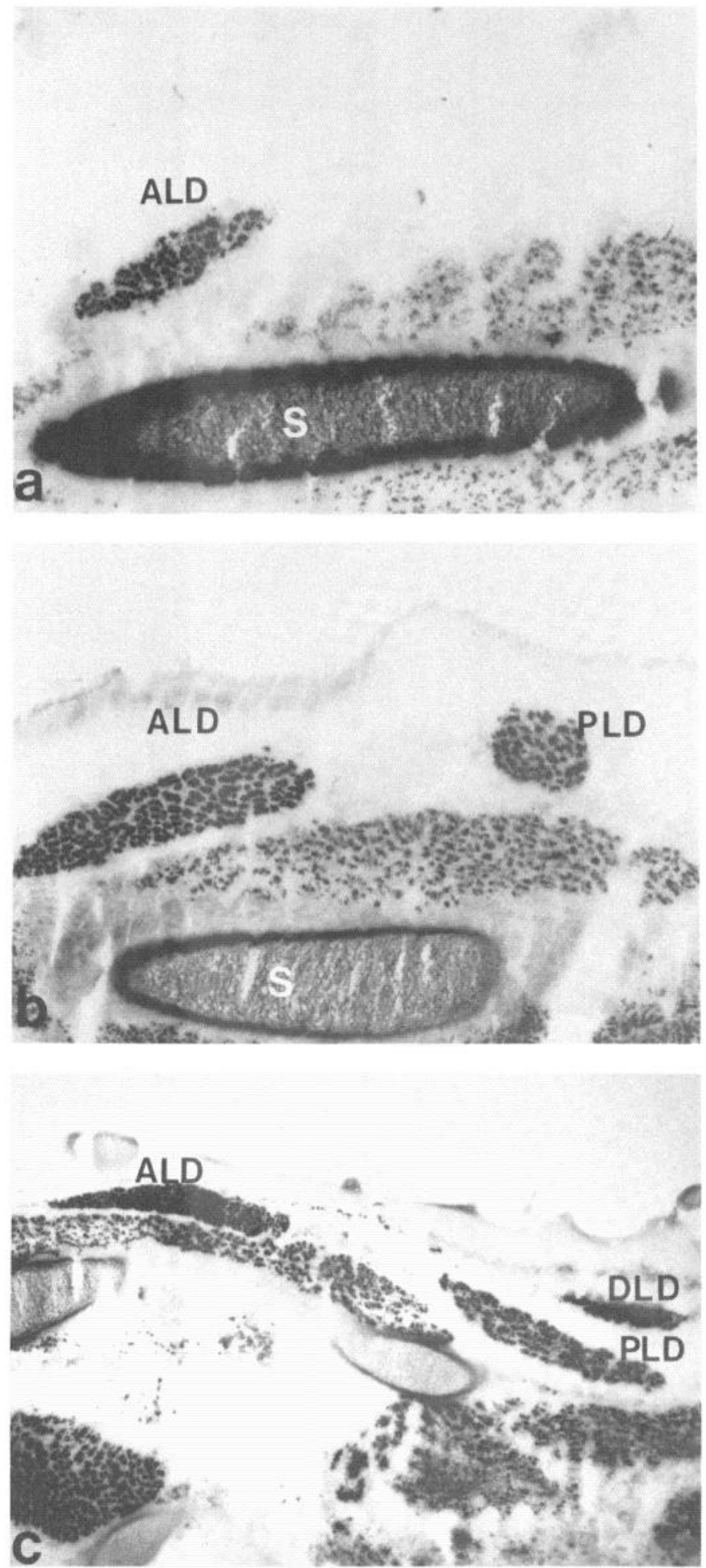

Figure 5. Photomicrographs of sections through the brachial region of St 35 ( 8 to 9 days) aneurogenic brachial muscles $(a)$ and stimulated aneurogenic muscles at St 35 (8 to 9 days) $(b)$ and St 37 (11 days) (c) to demonstrate alkali-stable myosin ATPase activity. The aneurogenic preparation shows the absence of the right PLD muscle whereas the right PLD and DLD muscles were rescued in the aneurogenic stimulated ones (cf. $a, b$, and $c$ ). $S$, scapula. Magnification, $a$ and $b: \times 64 ; c$ : $\times 40$.
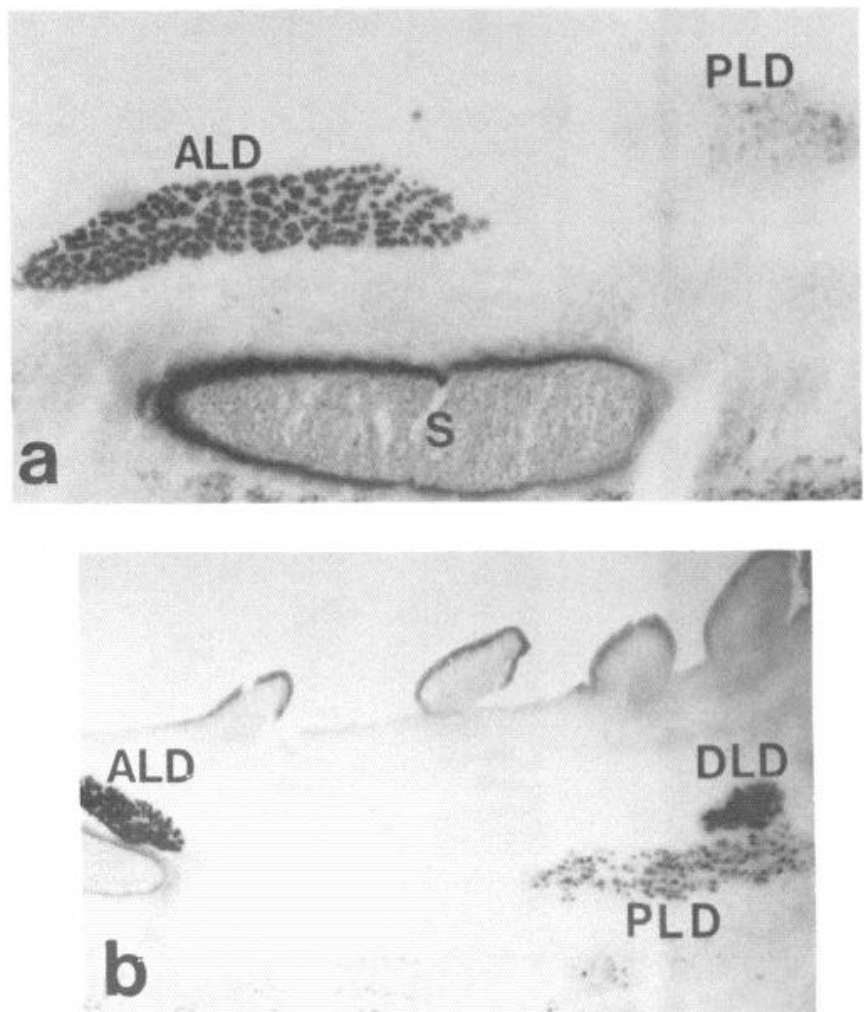

Figure 6. Photomicrographs of sections through the brachial region of St 35 ( 8 to 9 days) ( $a$ ) and St 37 (11 days) (b) stimulated aneurogenic muscles to demonstrate acid-stable myosin ATPase activity. A comparison of $a$ with its serial section in Figure $5 b$ illustrates the dual acidand alkali-stable myosin ATPase profile of the ALD muscle and the beginning of a few "atypical" PLD fibers, i.e., fibers with both acid and alkali stability. $b$, which is a slightly more dorsal section of the same embryo presented in Figure $5 c$, demonstrates the presence of the right ALD, DLD, and PLD muscles. Both the ALD and rescued DLD muscles exhibit dual acid and alkali stability (cf. $b$ and Fig. $5 c$ ); at this stage the "atypical" fibers within the PLD muscle are prominent. A similar profile characterizes innervated PLD muscles at this stage in ovo. $S$, scapula. Magnification, $a: \times 64 ; b: \times 40$.

preparations indicates that deletion of the brachial neural tube at day 2 in ovo did not interfere with its formation. Indeed, this operative procedure also did not prevent the formation of the vertebrae as well as the coracoid, humerus, radius, and ulna bones.

In addition to the unaltered profiles of the ALD, PLD, and DLD muscles, histochemical analyses indicated that the profiles of all stimulated aneurogenic brachial muscles derived from the primary dorsal and ventral muscle masses were similar to those of unstimulated aneurogenic and unoperated (innervated) embryos (see Table 1 in Butler et al., 1982a).

\section{Discussion}

Enhanced growth and survival of stimulated aneurogenic muscles. Deletion of the brachial neural tube of the chick embryo, performed at day 2 in ovo before the exit of peripheral nerves, does not interfere with the formation of brachial muscles but does impair their growth and survival. Thus, the aneurogenic model identifies a neural dependency for certain aspects of myogenesis during the period between initial nerve-muscle contact and the establishment of well characterized neuromuscular junctions. The present experiments were designed to isolate a specific role of peripheral nerves essential for both the growth and survival of muscles during this period. Our results demonstrated that experimentally applied activity augmented 
the growth of aneurogenic muscles and, indeed, rescued specific muscles which do not survive in the absence of neuronal influences. Chronic electrical stimulation via implanted electrodes was initiated at day 6 in ovo, the period when motility is first observed in innervated wings (for review, see Oppenheim, 1982; Cauwenbergs et al., 1983) and continued for periods ranging from 2 to 5 days. Volumetric analyses demonstrated that this stimulation regimen significantly enhanced growth of both the aneurogenic ALD and PLD muscles beyond levels characteristic of unstimulated aneurogenic muscles. This potent ability of activity to promote growth of embryonic muscles is emphasized if one considers that the aneurogenic ALD muscle was $50 \%$ smaller and the aneurogenic PLD muscle was $80 \%$ smaller than their innervated counterparts when stimulation was initiated. The positive response of the aneurogenic PLD muscle to stimulation was especially striking since this muscle does not survive beyond St 32 ( 7.5 day) in the absence of innervation. In fact, of the four muscles derived from the latissimus dorsi primordium, only the aneurogenic ALD muscle survived beyond this critical period. Following direct stimulation, the aneurogenic PLD, DLD, and MLD muscles all survived in the majority of embryos analyzed.

Our results that direct chronic electrical stimulation enhanced growth and survival of aneurogenic muscles implicate activity per se as an important growth-promoting influence during early myogenesis. First, activity alone was imposed upon embryonic myogenic cells denied both neurally mediated trophic and activity factors. Thus, we were able to monitor the effect of stimulation in the absence of trophic influences. Second, the stimulation regimen, initiated at day 6 in ovo, was effective prior to the period when neuromuscular junctions are established in the ALD and PLD (Atsumi, 1977) and other brachial (for review, see Bennett, 1983) muscles of unoperated embryos. Previously, experiments designed to investigate the role of neurally evoked activity on the growth of embryonic muscle focused either on the administration of pharmacological pre- and postsynaptic blocking agents ( $\alpha$-bungarotoxin, botulinum toxin, curare) during development early in ovo (Freeman et al., 1976; Laing, 1982; Bloom et al., 1983; McLennan, 1983) or on a comparison of surgically versus chemically denervated muscles (Bonner, 1978, 1980; Sohal and Holt, 1980; Harris, 1981; Creazzo and Sohal, 1983). The majority of these investigators concluded that growth of paralyzed embryonic muscles is more severely impaired during later stages of development. In fact, Harris (1981) and McLennan (1983) have indicated that impaired growth of paralyzed muscles is due primarily to the failure of secondary myotubes to form. To our knowledge, only the studies of Laing (1982), which blocked activity with $\alpha$-bungarotoxin, and Bonner (1978, 1980), which used a combined in vivo-in vitro analysis of clonable myoblasts after spinal cord cauterization or curarization, have demonstrated that the lack of neurally mediated muscle activity severely affects muscle growth during early stages of myogenesis. Their results are in agreement with the present observations that, prior to the formation of morphologically identified neuromuscular junctions, activity is an important growth-promoting influence.

Unaltered myosin ATPase profiles. Comparative histochemical analyses demonstrated that direct stimulation of aneurogenic brachial muscles did not alter myosin ATPase profiles. The specific profiles of individual brachial muscles were similar among stimulated aneurogenic, unstimulated aneurogenic, and innervated embryos at all periods analyzed, i.e., St 33 ( 7.5 to 8 days) to St 37 (11 days). These results contrast sharply with the abundant evidence that, during the postembryonic period, altered neural activity, achieved via direct or indirect electrical stimulation of intact or denervated muscles, alters the histochemical, biochemical, and physiological properties of muscles (Heilmann and Pette, 1980; Lømo et al., 1980; for review, see
Jolesz and Sreter, 1981; Klug et al., 1983). Thus, the ability of neural impulse activity to influence these muscle phenotypes may be acquired only after the formation of neuromuscular junctions. Evidence for this statement is derived from experiments whereby chronic spinal cord stimulation of intact chick embryos was performed between 10 and 15 days in ovo, after neuromuscular junctions have been morphologically observed (Atsumi, 1977). Under these stimulation conditions, PLD muscles examined during the last week of embryogenesis exhibited altered phenotypes (Renaud et al., 1978; 1983; Toutant et al., 1979, 1980).

Our previous analyses of unstimulated aneurogenic brachial muscles (Butler et al., 1982a) and our present investigation of stimulated aneurogenic muscles both demonstrate that the initial differentiation of specific muscle fiber types occurs independently of neuronal factors. Furthermore, the myosin ATPase profiles of embryonic avian brachial muscles innervated by foreign lumbosacral (Khaskiye et al., 1980; Laing and Lamb, 1983) or thoracic (Butler et al., 1982b; Cauwenbergs et al., 1983) nerves also remain unaltered.

Comments. Analysis of embryos subjected to deletion of appropriate segments of the neural tube reveal the extensive capacity of limbs for self-determination, as first demonstrated by Hamburger (1927). Although aneurogenic muscles of such embryos form initially, they fail to grow properly and, ultimately, to survive. In the present experiments, we have demonstrated that electrical stimulation of these aneurogenic muscles enhanced both their growth and survival, thus identifying activity as an important growth-promoting factor during early myogenesis. The mechanism whereby activity influences the growth and survival of aneurogenic muscles at the cellular and subcellular levels of analysis and the effect of other factors, such as neurotrophism (Oh and Markelonis, 1982), on aneurogenic muscle, remain to be elucidated.

\section{References}

Atsumi, S. (1977) Development of neuromuscular junctions of fast and slow muscles in the chick embryo: A light and electron microscopic study. J. Neurocytol. 6: 691-709.

Bennett, M. R. (1983) Development of neuromuscular synapses. Physiol. Rev. 63: 917-1048.

Bloom, J. W., E. Cosmos, and J. Butler (1983) Effects of activity dependent neural factors on muscle growth and maintenance in the young chick embryo. Soc. Neurosci. Abstr. 9: 8.

Bonner, P. H. (1978) Nerve-dependent changes in clonable myoblast populations. Dev. Biol. 66: 207-219.

Bonner, P. H. (1980) Differentiation of chick embryo myoblasts is transiently sensitive to functional denervation. Dev. Biol. 76: 79-86.

Butler, J., and E. Cosmos (1981a) Enzymic markers to identify musclenerve formation during embryogenesis: Mudified myosin ATPase and silver-cholinesterase histochemical reactions. Exp. Neurol. 73 . 831-836.

Butler, J., and E. Cosmos (1981b) Differentiation of the avian latissimus dorsi primordium: Analysis of fiber type expression using the myosin ATPase histochemical reaction. J. Exp. Zool. 218: 219-232.

Butler, J., J. Brierley, and E. Cosmos (1980) Aneural chick wings demonstrate histochemically normal myosin ATPase profiles. Am. Zool. 20: 805 .

Butler, J., E. Cosmos, and J. Brierley (1982a) Differentiation of muscle fiber types in aneurogenic brachial muscles of the chick embryo. J. Exp. Zool. 224: 65-80.

Butler, J., E. Cosmos, and J. Brierley (1982b) Response of brachial muscles to heterotopic innervation during embryogenesis. Can. J. Neurol. Sci. 9: 360 .

Castro, G. de O. (1963) Effects of reduction of nerve centers on the development of residual ganglia and on nerve patterns in the wing of the chick embryo. J. Exp. Zool. 152: 279-295.

Cauwenbergs, P., E. Cosmos, and J. Butler (1983) Alterations of wing motility and motoneuron number following heterotopic neural tube transplantation in chick embryos. Soc. Neurosci. Abstr. 9: 373. 
Close, R. (1972) Dynamic properties of mammalian skeletal muscles. Physiol. Rev. 52: 129-197.

Creazzo, T. L., and G. S. Sohal (1983) Neural control of embryonic acetylcholine receptor and skeletal muscle. Cell Tissue Res. 228: 112.

Freeman, S. S., A. G. Engel, and D. B. Drachman (1976) Experimental acetylcholine blockade of the neuromuscular junction. Effects on end plate and muscle fiber ultrastructure. Ann. N. Y. Acad. Sci. 274: 4659.

Hamburger, V. (1927) Entwicklungsphysiologische Beziehungen zwischen den Extremitäten der Amphibien und ihrer Innervation. Naturwissenschaften 15: 657-681.

Hamburger, V., and H. L. Hamilton (1951) A series of normal stages in the development of the chick embryo. J. Morphol. 88: 49-92.

Harris, A. J. (1981) Embryonic growth and innervation of rat skeletal muscles. I. Neural regulation of muscle fibre numbers. Philos. Trans. R. Soc. Lond. (Biol.) 293: 257-277.

Heilmann, C., and D. Pette (1980) Molecular transformations of sarcoplasmic reticulum in chronically stimulated fast-twitch muscles. In Plasticity of Muscle, D. Pette, ed., pp. 421-440, Walter de Gruyter $\&$ Co., Berlin.

Jolesz, F., and F. A. Sreter (1981) Development, innervation, and activity-pattern induced changes in skeletal muscle. Annu. Rev. Physiol. 43: 531-552.

Khaskiye, A., J. P. Toutant, M. Toutant, D. Renaud, and G. H. Le Douarin (1980) Effect of heterotopic innervation on the development of synaptic pattern in chick embryo muscles. Arch. Anat. Microsc. 69: $135-146$.

Klug, G., W. Wiehrer, H. Riechmann, E. Leberer, and D. Pette (1983) Relationships between early alterations in parvalbumins, sarcoplasmic reticulum and metabolic enzymes in chronically stimulated fast twitch muscle. Pflugers Arch. 399: 280-284.

Laing, N. G. (1982) Motor projection patterns to the hind limb of normal and paralysed chick embryos. J. Embryol. Exp. Morphol. 72: 269-286.

Laing, N. G., and A. H. Lamb (1983) The distribution of muscle fibre types in chick embryo wings transplanted to the pelvic region is normal. J. Embryol. Exp. Morphol. 78: 67-82.

Lømo, T., R. H. Westgaard, and L. Engebretsen (1980) Different stimulation patterns affect contractile properties of denervated rat soleus muscles. In Plasticity of Muscle, D. Pette, ed., pp. 297-309, Walter de Gruyter \& Co., Berlin
McArdle, J. J. (1983) Molecular aspects of the trophic influence of nerve on muscle. Prog. Neurobiol. 21: 135-198.

McLennan, I. S. (1983) Neural dependence and independence of myotube production in chicken hindlimb muscles. Dev. Biol. 98: 287294.

Oh, T. H., and G. J. Markelonis (1982) Chicken serum transferrin duplicates the myotrophic effects of sciatin on cultured muscle cells. J. Neurosci. Res. 8: 535-545.

Oppenheim, R. W. (1982) The neuroembryological study of behavior: Progress, problems, perspectives. Curr. Top. Dev. Biol. 17: 257-309.

Popiela, H. (1976) In vivo limb tissue development in the absence of nerves: A quantitative study. Exp. Neurol. 53: 214-226.

Popiela, H. (1977) In vivo limb muscle differentiation in the absence of nerves: A quantitative study. Exp. Neurol. 55: 160-172.

Renaud, D., G. H. Le Douarin, and A. Khaskiye (1978) Spinal cord stimulation in chick embryo: Effects on development of the posterior latissimus dorsi muscle and neuromuscular junctions. Exp. Neurol. 60: $189-200$

Renaud, D., M. -F. Gardahaut, T. Rouaud, and G. H. Le Douarin (1983) Influence of chronic spinal cord stimulation upon differentiation of $\beta$ muscle fibers in a fast muscle (posterior latissimus dorsi) of the chick embryo. Exp. Neurol. 80: 157-166.

Romanoff, A. L. (1960) The Avian Embryo. Structural and Functional Development, The Macmillan Co., New York.

Sohal, G. S., and R. K. Holt (1980) Role of innervation on the embryonic development of skeletal muscle. Cell Tissue Res. 210: 383393.

Sohal, G. S., and R. W. Wrenn (1984) Appearance of high-molecularweight acetylcholinesterase in aneural muscle developing in vivo. Dev. Biol. 101: 229-234.

Toutant, J. P., M. N. Toutant, D. Renaud, and G. H. Le Douarin (1979) Enzymatic differentiation of muscle fibre types in embryonic latissimus dorsi of the chick: Effects of spinal cord stimulation. Cell Differ. 8: $375-382$.

Toutant, M., J. -P. Bourgeois, J. -P. Toutant, D. Renaud, G. H. Le Douarin, and J. -P. Changeux (1980) Chronic stimulation of the spinal cord in developing chick embryo causes the differentiation of multiple clusters of acetylcholine receptor in the posterior latissimus dorsi muscle. Dev. Biol. 76: 384-395.

Tweedle, C. D., H. Popiela, and C. S. Thornton (1974) Ultrastructure of the development and subsequent breakdown of muscle in aneurogenic limbs (Amblystoma). J. Exp. Zool. 190: 155-166. 